\title{
Composition dependence of activation energy in solid phase epitaxial growth of $\mathrm{Si}_{1-x} \mathrm{Ge}_{x}$ alloys
}

\author{
K. Y. Suh and Hong H. Lee ${ }^{\text {a) }}$ \\ Department of Chemical Engineering, Seoul National University, Seoul 151-742, Korea
}

(Received 27 March 1996; accepted for publication 28 August 1996)

\begin{abstract}
A theoretical model called the "multibody model" is developed for the composition dependence of the activation energy. The model that is based on the diffusion required of the recrystallization for the solid phase epitaxy does not involve any adjustable parameters and is shown to represent experimental data satisfactorily. For the $\mathrm{Si}_{1-x} \mathrm{Ge}_{x}$ alloys that are of diamond structure, the most logical choice is the "five-body model" involving five atoms. The model is equally applicable to any other binary alloy. (C) 1996 American Institute of Physics. [S0021-8979(96)06123-3]
\end{abstract}

\section{INTRODUCTION}

Heterostructures of $\mathrm{Si}_{1-x} \mathrm{Ge}_{x}$ have drawn considerable interest because of new capabilities that they can offer. Their usage has been demonstrated in high performance bipolar devices and a wide variety of novel electronic devices. ${ }^{1,2}$ One way of forming the heterostructures is solid phase epitaxy in which an amorphous film of $\mathrm{Si}_{1-x} \mathrm{Ge}_{x}$ on $\mathrm{Si}$ is recrystallized.

Typical experimental studies of the solid phase epitaxy involve depositing a film of $\mathrm{Si}_{1-x} \mathrm{Ge}_{x}$ epilayer on a Si substrate, amorphizing from the free surface to a certain depth and then annealing so that the amorphized layer can regrow epitaxially on the underlying substrate. When only the $\mathrm{Si}_{1-x} \mathrm{Ge}_{x}$ epilayer is amorphized, the solid phase epitaxy (SPE) is termed unstrained or stress-relaxed SPE, whereas it is called strained SPE when the underlying $\mathrm{Si}$ is also amorphized.

There are at present two proposed models for the SPE mechanism. These are the dangling bond $\operatorname{model}^{3}$ and the diffusion model. ${ }^{4,5}$ A rather complete comparison of the two models is given by Lu et al. ${ }^{6}$

Despite the significant advances made in the SPE, there still exists an anomaly that either model cannot explain with regard to the composition dependence of the activation energy of the growth. As the composition $x$ in $\mathrm{Si}_{1-x} \mathrm{Ge}_{x}$ increases, the activation energy goes through a maximum instead of showing a monotonic change from pure silicon to pure germanium.

In this paper, we present a theoretical model that can explain quantitatively the composition dependence in an $a$ priori manner.

\section{THEORETICAL MODEL}

The activation energies of the SPE growth for both pure silicon and pure germanium are rather well established. The crystallization rate of pure silicon has been measured over a wide temperature range $\left(500-650^{\circ} \mathrm{C}\right)$ and shown to be a thermally activated process characterized by an activation energy of 2.6-2.7 eV., The fact that the activation energy is almost the same over several orders of magnitude implies

a) Author to whom correspondence should be addressed. that a single rate-limiting step may control the recrystallization rate at all temperatures. To the extent the experimental data are available for $\mathrm{Ge}$, the activation energy shows a similar trend as for silicon but with an activation energy of 2.0$2.2 \mathrm{eV}$ (Refs. 4, 7, and 8) in the temperature range of 300$450{ }^{\circ} \mathrm{C}$.

There are a number of studies ${ }^{7-11}$ reporting the experimentally determined values of the activation energies for $\mathrm{Si}_{1-x} \mathrm{Ge}_{x}$ on $\mathrm{Si}$. To the contrary of what one might expect, the activation energies, when the composition $x$ in $\mathrm{Si}_{1-x} \mathrm{Ge}_{x}$ is less than 0.4, are larger than that for pure silicon in both strained and unstrained SPE cases, decreasing thereafter to that for pure germanium. In general, the regrowth rate of stressed alloys is somewhat less than that of pure silicon, while it is larger for stress-relaxed alloys.

The activation process, according to the dangling bond model, ${ }^{3}$ is viewed as one in which a bond-breaking event is responsible for the observable activation energy for regrowth. The diffusion model, ${ }^{4,5}$ on the other hand, views the regrowth as a consequence of mass transport from near the recrystallizing interface to the crystalline phase, the activation energy being that required for the vacancy formation and migration at the interface. However, neither model can describe quantitatively the anomalously large activation energies for Si-rich alloys.

Either model can be used as a starting point for determining the composition dependence of the activation energy. In the dangling bond model, however, the activation energy is expressed as the sum of dangling bond formation and migration energies, or $\Delta H=\Delta H_{f}+\Delta H_{m}$. Since $\Delta H_{f} \gg \Delta H_{m}$, the dangling bond formation energy, $\Delta H_{f}$, determines the activation energy. The bonding energies of $\mathrm{Si}-\mathrm{Si}, \mathrm{Si}-\mathrm{Ge}$, and $\mathrm{Ge}-\mathrm{Ge}$ are in the order of $\mathrm{Si}-\mathrm{Si}, \mathrm{Si}-\mathrm{Ge}$, and $\mathrm{Ge}-\mathrm{Ge}$ so that the activation energy of $\mathrm{Si}-\mathrm{Ge}$ should lie somewhere between the values of $\mathrm{Si}-\mathrm{Si}$ and $\mathrm{Ge}-\mathrm{Ge}$. Hence, the dangling bond model cannot be used as a basis for explaining the anomalously large activation energy of silicon rich $\mathrm{Si}_{1-x} \mathrm{Ge}_{x}$. Therefore, we base our model on the diffusion process.

The recrystallization in SPE requires movement of atoms. The atoms near the amorphous-crystal interface diffuse into crystal vacancies. In the process, the diffusing atoms can encounter various types of atom configuration at the interface. The atom configuration may involve many atoms. 


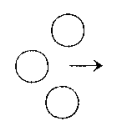

$\mathrm{E}_{1}$

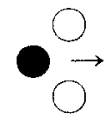

$\mathrm{E}_{4}$

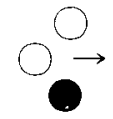

$\mathrm{E}_{2}$

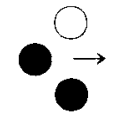

$\mathrm{E}_{5}$

(a)

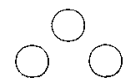

4.1eV

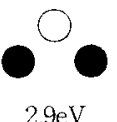

$2.9 \mathrm{eV}$

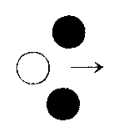

$\mathrm{E}_{3}$

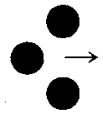

$\mathrm{E}_{6}$

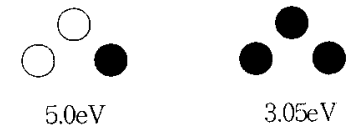

(b)

: silicon

germanium

FIG. 1. (a) Six configurations considering two neighboring atoms and one diffusing atom. (b) Three-body model supposing that only vacancy formation energy is needed for solid phase epitaxy.

However, the simplest are the ones involving two atoms as shown in Fig. 1(a). The two atom configurations that one diffusing $\mathrm{Si}$ atom encounters are the first three in Fig. 1(a) and those that one diffusing Ge atom encounters are the bottom three. Let $E_{i}(i=1,2, \ldots, 6)$ be the diffusion activation energy for the six configurations numbered 1-6 from left to right so that $E_{1}$, for instance, is the activation energy for silicon to diffuse into silicon ( $\mathrm{Si}$ self-diffusion). Any diffusing atom, whether it is silicon or germanium, can encounter any of the six configurations whatever the composition $x$ may be. The probability that a diffusing atom encounters a certain configuration is given by the composition so that the probability for the first configuration in Fig. 1(a) is $(1-x)^{3}$, that for the second is $2 x(1-x)^{2}$, and so on.

It follows then that the activation energy for the alloy $\mathrm{Si}_{1-x} \mathrm{Ge}_{x}$ can be written as

$$
\begin{aligned}
E_{\mathrm{Si}_{1-x} \mathrm{Ge}_{x}}= & k\left[(1-x)^{3} E_{1}+2(1-x)^{2} x E_{2}+(1-x) x^{2} E_{3}\right. \\
& \left.+(1-x)^{2} x E_{4}+2(1-x) x^{2} E_{5}+x^{3} E_{6}\right],
\end{aligned}
$$

where $k$ is a constant factor that accounts for the difference between bulk diffusion that is typical of diffusion experiments and the diffusion from near an interface to an interface crystal structure. Only a fraction of the bulk diffusion activation energy would be required for the diffusion relevant to the recrystallization. According to theoretical treatments of the diamond lattice structure ${ }^{12,13}$ and experimental work, ${ }^{14-16}$ about two-thirds of the total activation energy is required for vacancy formation for diffusion by a vacancy-migration mechanism while the remaining one third is expended as enthalpy of vacancy migration. If it is assumed that only vacancy formation is necessary for the diffusion from near the interface to the interface crystal structure, the value of $k$ could be taken as $2 / 3$, yielding

$$
\begin{aligned}
E_{\mathrm{Si}_{1-x} \mathrm{Ge}_{x}=} & \frac{2}{3}\left[(1-x)^{3} E_{1}+2(1-x)^{2} x E_{2}+(1-x) x^{2} E_{3}\right. \\
& \left.+(1-x)^{2} x E_{4}+2(1-x) x^{2} E_{5}+x^{3} E_{6}\right] .
\end{aligned}
$$

There are a number of studies ${ }^{17-22}$ reported in the literature on the bulk diffusion of silicon in silicon $(\mathrm{Si} \rightarrow \mathrm{Si})$, germanium, in silicon $(\mathrm{Ge} \rightarrow \mathrm{Si})$, silicon in germanium $(\mathrm{Si} \rightarrow \mathrm{Ge})$, and germanium self-diffusion $(\mathrm{Ge} \rightarrow \mathrm{Ge})$. The activation energy for the silicon self-diffusion ranges from 3.95 to 5.42 eV. ${ }^{17,22}$ It depends on the temperature at which the diffusion experiments are carried out and the methods by which the diffusion parameters are measured. For the germanium selfdiffusion, the activation energy ranges from 2.95 to 3.14 eV. ${ }^{17,19}$ For silicon diffusion in germanium, the activation energy reported is $2.9 \mathrm{eV},{ }^{17}$ whereas that for germanium diffusion in silicon ranges from 4.7 to $5.3 \mathrm{eV} .^{17,20,21}$

A few questions need to be resolved before we can assign specific values to $E_{i}$. There is a large scatter in the reported value of the activation energy for the silicon selfdiffusion $\left(E_{1}\right)$, ranging from 3.95 to $5.42 \mathrm{eV}$. Hirvonen and Anttila ${ }^{18}$ attributed the scatter to experimental uncertainty and the corresponding errors caused by short annealing times associated with the conventional radio-tracer method due to a relatively short half-life of ${ }^{31} \mathrm{Si}$ isotope that is used in the method. To alleviate the experimental problem, they used a $(p, \gamma)$ resonance broadening method that allows long annealing times and arrived at an activation energy of $4.1 \mathrm{eV}$. This energy is used for $E_{1}$. There is only one activation energy reported for silicon diffusion in germanium $\left(E_{3}\right)$ and this value $(2.9 \mathrm{eV})$ is used for $E_{3}$. It is noted in this regard that the SPE experiments are carried out at temperatures below $650{ }^{\circ} \mathrm{C}$ whereas the self-diffusion experiments are performed at temperatures higher than $900{ }^{\circ} \mathrm{C}$, typically above $1100{ }^{\circ} \mathrm{C}$. The ranges of the activation energies for the other cases are relatively small such that an average value may be used for each: $E_{4}(\mathrm{Ge} \rightarrow \mathrm{Si})=5.0 \mathrm{eV}$ and $E_{6}(\mathrm{Ge} \rightarrow \mathrm{Ge})=3.05 \mathrm{eV}$. In the absence of detailed information for $E_{2}$, it may be taken to be the same as $E_{4}$ since both involve the same atom configuration. By the same reasoning, $E_{5}$ may be set equal to $E_{3}$. It is noted in this regard that $E_{2}$ and $E_{5}$ cannot be determined by the usual experimental technique. Then, Eq. (2) reduces to

$$
\begin{aligned}
E_{\mathrm{Si}_{1-x} \mathrm{Ge}_{x}}= & \frac{2}{3}\left[(1-x)^{3} E_{1}+3(1-x) x^{2} E_{3}+3(1-x)^{2} x E_{4}\right. \\
& \left.+x^{3} E_{6}\right]
\end{aligned}
$$

where $E_{1}=4.1 \mathrm{eV}, E_{3}=2.9 \mathrm{eV}, E_{4}=5.0 \mathrm{eV}$, and $E_{6}=3.05$ $\mathrm{eV}$.

The probabilities and reasonings are such that the atom configurations shown in Fig. 1(a) reduce to those in Fig. 1(b). The corresponding activation energies are given at the bottom. Since three atoms are involved, the model may be called the "three-body model", for which the activation energy is given by

$$
\begin{aligned}
E_{\mathrm{Si}_{1-x} \mathrm{Ge}_{x}}= & (1-x)^{3} 2.73+3(1-x) x^{2} 1.93 \\
& +3(1-x)^{2} \times 3.33+x^{3} 2.03
\end{aligned}
$$




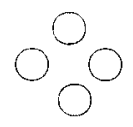

$4.1 \mathrm{eV}$

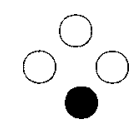

$2.9 \mathrm{eV}$

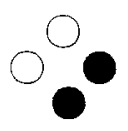

$3.95 \mathrm{eV}$

$5.0 \mathrm{eV}$

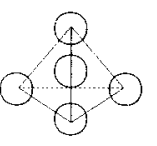

$4.1 \mathrm{eV}$

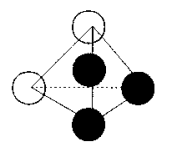

$3.6 \mathrm{eV}$

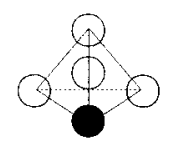

$5.0 \mathrm{eV}$

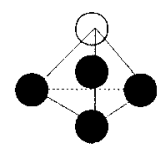

$2.9 \mathrm{eV}$

(b)

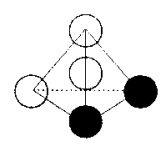

$4.3 \mathrm{eV}$

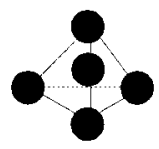

$3.05 \mathrm{eV}$
FIG. 2. (a) Four-body model. Activation energy for the middle case is simply taken as the average of the two adjacent ones or $3.95=(2.9+5.0) / 2$. (b) Five-body model. Weighted average is used for activation energies for the two middle configuration or $2.86=(2 \times 5.0+2.9) / 3,2.4=(5.0+2 \times 2.9) / 3$.

As indicated at the outset, the simplest possible case is that shown in Fig. 1(b) involving three atoms. If four atom configurations are considered, the "four-body model", shown in Fig. 2(a) results. The activation energy for the middle case in the figure is simply taken as the average of the two adjacent ones. It follows then that the four-body model yields

$$
\begin{aligned}
E_{\mathrm{Si}_{1-x} \mathrm{Ge}_{x}}= & (1-x)^{4} 2.73+4(1-x) x^{3} 1.93+6(1-x)^{2} x^{2} 2.63 \\
& +4(1-x)^{3} x 3.33+x^{4} 2.03
\end{aligned}
$$

Perhaps the most logical choice is the five-body model shown in Fig. 2(b) for the diamond structure being considered. The five-body represents the five atoms forming the tetrahedron, which is one-eighth of the unit cell of the diamond crystal structure. As shown in Fig. 2(b), weighted averages are used for the activation energies for the two middle configurations. For the five-body model, the activation energy is given by

$$
\begin{aligned}
E_{\mathrm{Si}_{1-x} \mathrm{Ge}_{x}}= & (1-x)^{5} 2.73+5(1-x) x^{4} 1.93 \\
& +10(1-x)^{3} x^{2} 2.86+10(1-x)^{2} x^{3} 2.4 \\
& +5(1-x)^{4} x 3.33+x^{5} 2.03 .
\end{aligned}
$$

\section{COMPARISON WITH EXPERIMENT AND DISCUSSION}

The theoretical model results can be compared with the experimental data reported in the literature. ${ }^{7-11}$ The experimental activation energy is obtained by plotting the loga-

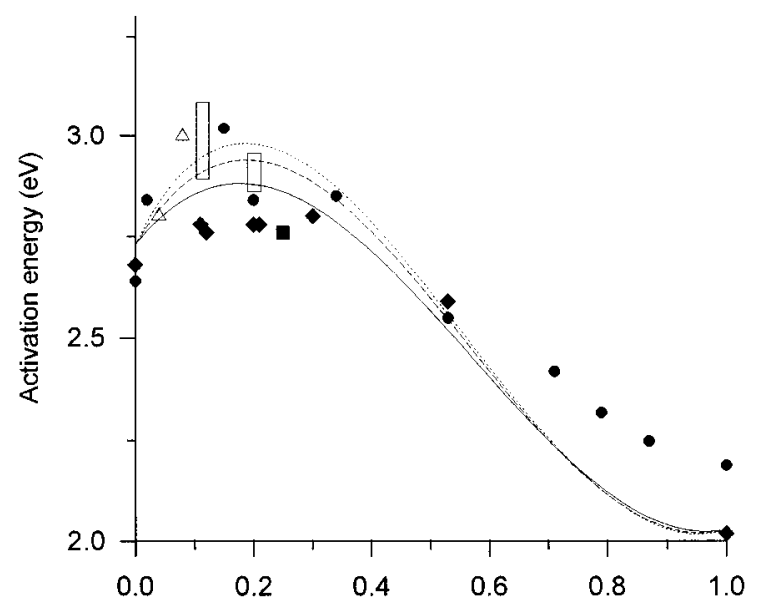

Composition of $\mathrm{Ge}\left(\mathrm{x}\right.$ in $\left.\mathrm{Si}_{1-\mathrm{x}} \mathrm{Ge}_{\mathrm{x}}\right)$

FIG. 3. Comparison of the multibody model with experimental data. The data for relaxed alloys are taken from Ref. 7 (filled circles), Ref. 8 (filled diamonds), and Ref. 9 (filled squares). The data for some stained layers are also shown (unfilled triangles from Ref. 10, unfilled diamonds from Ref. 11). The curves show the theoretical activation energy as determined by the multibody model: three-body model (solid), four-body model (dashed), and five-body model (dotted).

rithm of the SPE rate against the inverse of temperature, the slope of which leads to the activation energy. The comparison is shown in Fig. 3 for both the relaxed alloys (filled symbols) and the strained alloys (unfilled symbols).

The comparison is seen to be satisfactory. As can be seen from Fig. 3, the peak gets higher and the shape becomes somewhat broader as one goes from the three-body model (solid curve) to the five-body model (dotted curve).

Although no adjustable parameters are needed in representing the composition dependence of the activation energy, one may choose one parameter in the four-body model and two parameters in the five-body model, particularly in view of the uncertainty involved in using an average value in the four-body model and two weighted averages in the five-body model. Considering that the experimental data in Fig. 3 are from five different sources and there is experimental uncertainty in determining the activation energy, it is not clear whether making these parameters adjustable is justified. Nevertheless, it might be desirable to use the adjustable parameters to represent a set of data from the same experiment (source).

The multibody model can easily be extended to any other binary alloys $A_{1-x} B_{x}$ in the following general form:

$$
E_{A_{1-x} B_{x}}=k \sum_{i} f_{i}(x) E_{i}
$$

where $f_{i}(x)$ is the probability of encounter with an $i$ type of atom configuration, and $E_{i}$ is the corresponding activation energy for the bulk diffusion. The most logical choice of the number of atoms involved is that corresponding to the subunit of the unit cell of the alloy crystal structure. 
${ }^{1}$ G. L. Patton, J. H. Comfort, B. S. Meyerson, E. F. Crabbe, G. J. Scilla, E. D. Fresart, J. M. C. Stork, J. Y. C. Sun, D. L. Harame, and J. N. Burghartz, IEEE Electron Device Lett. 11, 171 (1990).

${ }^{2}$ C. A. King, J. L. Hoyt, C. M. Gronet, J. F. Gibbons, M. P. Scott, and J. A. Turner, IEEE Electron. Device Lett. 10, 52 (1989).

${ }^{3}$ F. Spaepen and D. Turnbull, in Laser-Solid Interactions and Laser Processing, edited by S. D. Ferris, H. J. Leamy, and J. M. Poate, AIP Conf. Proc. No. 50 (AIP, New York, 1979), p. 73.

${ }^{4}$ L. Csepregi, R. P. Küllen, J. W. Mayer, and T. W. Sigmon, Solid State Commun. 21, 1019 (1977).

${ }^{5}$ I. Suni, G. Goltz, M. A. Nicolet, and S. S. Lau, Thin Solid Films 93, 171 (1982).

${ }^{6}$ Guo-Quan Lu, Eric Nygren, and Michael J. Aziz, J. Appl. Phys. 70, 5323 (1991).

${ }^{7}$ T. E. Haynes, M. J. Antonell, C. Archie Lee, and K. S. Jones, Phys. Rev. B 51, 7762 (1995).

${ }^{8}$ R. G. Elliman, W. C. Wong, and P. Kringhøj, in Crystallization and Related Phenomena in Amorphous Materials, edited by M. Libera, T. E. Haynes, P. Cebe, and J. E. Dickinson, Jr., MRS Symposia Proceedings No. 321 (Materials Research Society, Pittsburgh, 1994), p. 375;
${ }^{9}$ P. Kring $\varnothing j$ and R. G. Elliman, Phys. Rev. Lett. 73, 858 (1994).

${ }^{10}$ S. Y. Shiryaev, M. Fyhn, and A. Nylandsted Larson, Appl. Phys. Lett. 63, 3476 (1993)

${ }^{11}$ Q. Z. Hong, J. G. Zhu, and J. W. Mayer, J. Appl. Phys. 71, 1768 (1992).

${ }^{12}$ C. Lee, T. E. Haynes, and K. S. Jones, Appl. Phys. Lett. 62, 501 (1993).

${ }^{13}$ R. A. Swalin, J. Phys. Chem. Solids 18, 290 (1961).

${ }^{14}$ K. H. Benneman, Phys. Rev. 137, 1497 (1965).

${ }^{15}$ W. J. Armstrong, J. Electrochem. Soc. 109, 1065 (1962).

${ }^{16}$ R. A. Logan, Phys. Rev. 101, 1455 (1956)

${ }^{17}$ S. Mayburg, Phys. Rev. 95, 38 (1954).

${ }^{18}$ J. Räisänen, J. Hirvonen, and A. Anttila, Solid-State Electron. 24, 333 (1981).

${ }^{19}$ J. Hirvonen and A. Anttila, Appl. Phys. Lett. 35, 703 (1979).

${ }^{20}$ M. Werner, H. Mehrer, and H. D. Hochheimer, Phy. Rev. 32, 3930 (1985).

${ }^{21}$ G. L. McVay and A. R. DuCharme, J. Appl. Phys. 44, 1409 (1973).

${ }^{22}$ D. A. Petrov, Yu. M. Shaskov, and I. P. Akimchenko, Yop. Met. fiz. Poluprov. Akad. Nauk SSSR Tr. Vtorogo Soveshch. Moscow 130 (1957).

${ }^{23}$ J. M. Fairfield and B. J. Masters, J. Appl. Phys. 38, 3148 (1967). 